\title{
Effects of Polyelectrolytes on the Diffusion of Low Molecular Weight Ionic Solutes
}

\author{
Mohamed Z. El-A BD, Ahmed A. Zatout, Gomaa H. SEDAhMed, \\ and Ahmed H. NEGM \\ Chemical Engineering Department, Faculty of Engineering, \\ Alexandria University, Alexandria, Egypt
}

(Received October 3, 1987)

\begin{abstract}
The diffusion of sodium sulphate in dilute solutions of carboxymethylcellulose sodium salt $(\mathrm{CMC})$ was studied using the porous frit technique. It was found that at relatively low CMC concentrations, the diffusivity of sodium sulphate $(D)$ is higher than that in water $\left(D_{0}\right)$; with increasing CMC concentration, the diffusivity of sodium sulphate decreases gradually and reaches a value lower than that in water. The relation between salt diffusivity ratio $\left(D / D_{0}\right)$ and $C M C$ concentration $(C)$ is represented by the equation: $D / D_{0}=a-b C$. The effect of temperature on the diffusivity of sodium sulphate in CMC solutions was found to obey the Arrhenius equation with an activation energy of $2600 \mathrm{cal} \mathrm{mol}^{-1}$.

KEY WORDS Diffusion / Polyelectrolytes / Carboxymethylcellulose / Sodium Sulphate / Electrolytes /
\end{abstract}

The diffusive transport of low molecular weight solutes in polymeric solutions is of a great interest not only in the chemical industry but also in biological processes. Although several theories ${ }^{1-3}$ have been introduced to account for the diffusion of low molecular weight solutes in polymer solutions, none was found to have general validity. Most previous studies have concentrated on the diffusion of nonelectrolytes in nonionic polymers. ${ }^{1-4}$ The object of the present work is to shed some light on the diffusion of low molecular weight solutes in dilute polyelectrolytes such as carboxymethylcellulose sodium salt (CMC) which was chosen to conduct the present study in view of its technical importance. Since its commercial introduction in 1944, CMC has found use in an increasing and wide variety of industries such as adhesives, cosmetics, coatings, food technology, paper industry, pharmaceuticals and textile industry. The most important functions of $\mathrm{CMC}$ in these industries are thickening, suspending, stabilizing and film forming. In all these applications CMC has to be mixed with low molecular solutes. The rate of dissolution of these solutes in CMC solution depends among other factors on the diffusivity of these solutes in $\mathrm{CMC}$; i.e., to predict the rate of solute dissolution in CMC solution, the solute diffusivity in CMC solution should be known. Although some works have been done on the diffusion of nonionic low molecular weight solutes in $\mathrm{CMC}^{3,4}$ little has been done on the diffusion of electrolytes in CMC. The present work is concerned with the diffusion of electrolytes such as sodium sulphate in CMC. To this end the porous frit technique, which has been used for studying salt diffusion, was chosen to conduct the present study in view of its rapidity, simplicity and accuracy particularly at high salt concentration.

\section{EXPERIMENTAL TECHNIQUE}

A frit of unglazed porcelain of $230 \mathrm{~g}$ dry weight, $10 \mathrm{~mm}$ thickness and $7 \mathrm{~cm}$ in radius 
which has been soaked with sodium sulphate for 24 hours was suspended in a 301 thermostated CMC solution from one arm of an analytical balance and the apparent weight of the disc $(w)$ was measured as a function of time $(t)$. The slope of the line obtained by plotting $\log w$ versus $t$ is related to the diffusion coefficient of the salt $(D)$ by the equation: ${ }^{5}$

$$
D=-K \times \text { slope }
$$

The frit constant $K$ was determined by carrying out diffusion experiments on one molar aqueous potassium chloride whose diffusion coefficient is $1.89 \times 10^{-5} \mathrm{~cm}^{2} \mathrm{~s}^{-1}$ at $25^{\circ} \mathrm{C}^{10}$ For the four frits used in the present study the disc constants were $7.05 \times 10^{-3}, 8.4 \times 10^{-3}$, $7.5 \times 10^{-3}$, and $8.1 \times 10^{-3}$. Diffusion of so- dium sulphate in the concentration range $0.493-0.99 \mathrm{M}$ was measured in water and CMC solutions which ranged in concentration from 2 to $20 \mathrm{gl}^{-1}$. CMC used in the present work (CMC-7 1) was provided by Hercules; its molecular weight was 80000 , degree of polymerization 400 , and degree of substitution 0.7 . Rheological measurements showed all CMC solutions used to be Newtonian. Viscosity used to calculate the Einstein-Stokes ratio was determined by means of an Ostwalds viscometer.

\section{RESULTS AND DISCUSSION}

Figure 1 is typical $\log w$ vs. time used to obtain the diffusion coefficient of sodium sulphate under different conditions using equa-

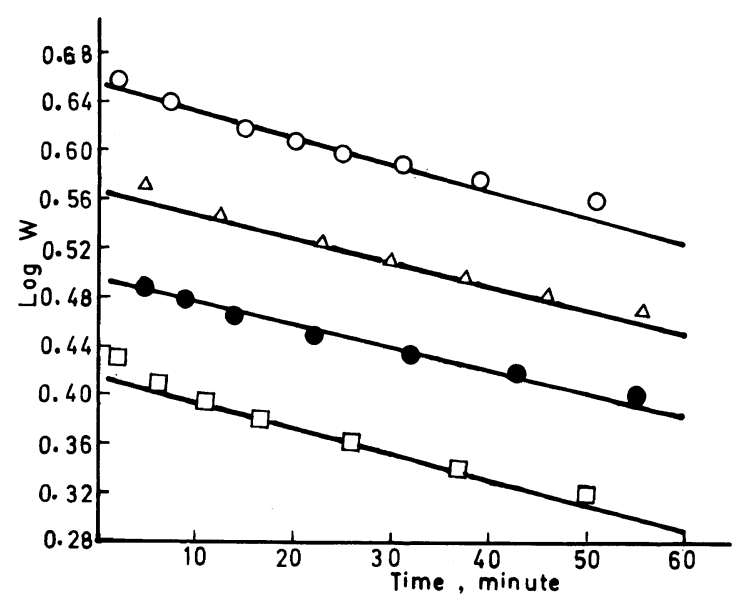

Figure 1. $\log w$ vs. time for the diffusion of $0.99 \mathrm{M}$ sodium sulphate at $25^{\circ} \mathrm{C} . \mathrm{Na}_{2} \mathrm{SO}_{4}$ concentration $=$ $0.99 \mathrm{M}$; temperature $=25^{\circ} \mathrm{C}, \mathrm{CMC}$ concentration: $\bigcirc, 2 \mathrm{gl}^{-1} ; \mathrm{O}, 4 \mathrm{gl}^{-1} ; \triangle, 6 \mathrm{gl}^{-1} ; \square, 8 \mathrm{gl}^{-1}$

Table I. Effect of CMC concentration on the diffusivity of $\mathrm{Na}_{2} \mathrm{SO}_{4}$ at $25^{\circ} \mathrm{C}$

\begin{tabular}{|c|c|c|c|c|c|c|c|c|c|c|c|}
\hline \multirow{2}{*}{$\begin{array}{c}\mathrm{Na}_{2} \mathrm{SO}_{4} \\
\text { Concent- } \\
\text { ration }\end{array}$} & \multicolumn{11}{|c|}{ Diffusivity of $\mathrm{Na}_{2} \mathrm{SO}_{4}$ at different CMC concentrations $\times 10^{5} / \mathrm{cm} \mathrm{s}^{-1}$} \\
\hline & \multirow{2}{*}{$\begin{array}{l}\text { Pure } \\
\text { water }\end{array}$} & \multirow{2}{*}{$\begin{array}{l}2 \mathrm{gl}^{-1} \\
\mathrm{CMC}\end{array}$} & \multirow{2}{*}{$\begin{array}{l}4 \mathrm{gl}^{-1} \\
\mathrm{CMC}\end{array}$} & \multirow{2}{*}{$\begin{array}{l}6 \mathrm{gl}^{-1} \\
\mathrm{CMC}\end{array}$} & \multirow{2}{*}{$\begin{array}{l}8 \mathrm{gl}^{-1} \\
\mathrm{CMC}\end{array}$} & \multirow{2}{*}{$\begin{array}{c}10 \mathrm{gl}^{-1} \\
\mathrm{CMC}\end{array}$} & \multirow{2}{*}{$\begin{array}{c}12 \mathrm{gl}^{-1} \\
\mathrm{CMC}\end{array}$} & \multirow{2}{*}{$\begin{array}{c}14 \mathrm{gl}^{-1} \\
\mathrm{CMC}\end{array}$} & \multirow{2}{*}{$\begin{array}{c}16 \mathrm{gl}^{-1} \\
\mathrm{CMC}\end{array}$} & \multirow{2}{*}{$\begin{array}{c}18 \mathrm{gl}^{-1} \\
\mathrm{CMC}\end{array}$} & \multirow{2}{*}{$\begin{array}{c}20 \mathrm{gl}^{-1} \\
\mathrm{CMC}\end{array}$} \\
\hline $\mathbf{M}$ & & & & & & & & & & & \\
\hline 0.493 & 1.33 & 1.54 & 1.47 & 1.42 & 1.35 & 1.3 & 1.24 & 1.18 & 1.12 & 1.06 & 0.95 \\
\hline 0.634 & 1.36 & 1.58 & 1.53 & 1.47 & 1.40 & 1.35 & 1.30 & 1.24 & 1.17 & 1.12 & 1.04 \\
\hline 0.775 & 1.39 & 1.62 & 1.57 & 1.51 & 1.45 & 1.40 & 1.35 & 1.29 & 1.23 & 1.16 & 1.12 \\
\hline 0.990 & 1.42 & 1.68 & 1.63 & 1.57 & 1.52 & 1.46 & 1.40 & 1.36 & 1.20 & 1.22 & 1.16 \\
\hline
\end{tabular}


tion 1. Table I and Figure 2 show the effects of CMC concentration on the diffusivity and diffusivity ratio of sodium sulphate, respectively. At relatively low CMC concentrations the diffusivity of sodium sulphate is higher

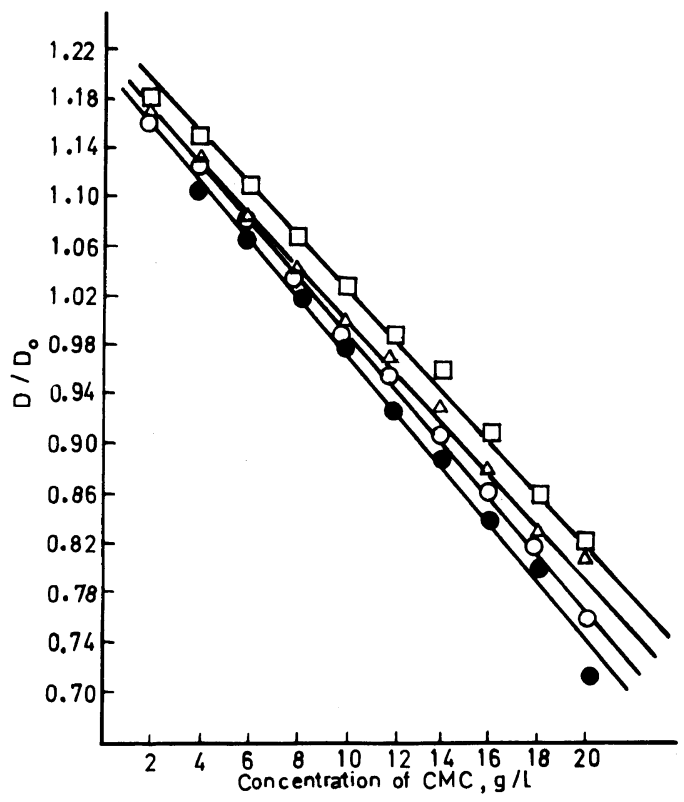

Figure 2. Effect of CMC concentration on the diffusivity ratio of sodium sulphate; temperature $25^{\circ} \mathrm{C}$. $\mathrm{Na}_{2} \mathrm{SO}_{4}$ concentration: $0,0.493 \mathrm{M} ; 0,0.634 \mathrm{M} ; \triangle$, $0.775 \mathrm{M} ; \square, 0.990 \mathrm{M}$. than that in water; with increasing CMC concentration the diffusivity decreases gradually and reaches a value lower than that in water. The relation between the diffusivity ratio $\left(D / D_{0}\right)$ and CMC concentration $(C)$ can be represented by the equation.

$$
D / D_{0}=a-b C
$$

To explain the diffusion behaviour of sodium sulphate in CMC solution, the properties of $\mathrm{CMC}$ as a macromolecule and as a polyelectrolyte should be considered. CMC exerts the following effects on the diffusing salt:

i) As a polyelectrolyte. CMC ionized in solution to produce $\mathrm{Na}^{+}$and negatively charged polymer ion. Interionic attractions take place between the diffusing salt ions and the polymer ions with consequent decrease in the activity of the diffusing salt ions and its chemical potential ${ }^{10}$; this gives rise to a decrease in salt diffusivity.

ii) The presence of CMC in solution increases the viscosity with consequent decrease in salt diffusivity. The ability of CMC to increase solution viscosity depends on the degree of expansion of the polymer molecules. ${ }^{11}$ A highly stretched polymer molecule leads to a large increase in solution viscosity while a

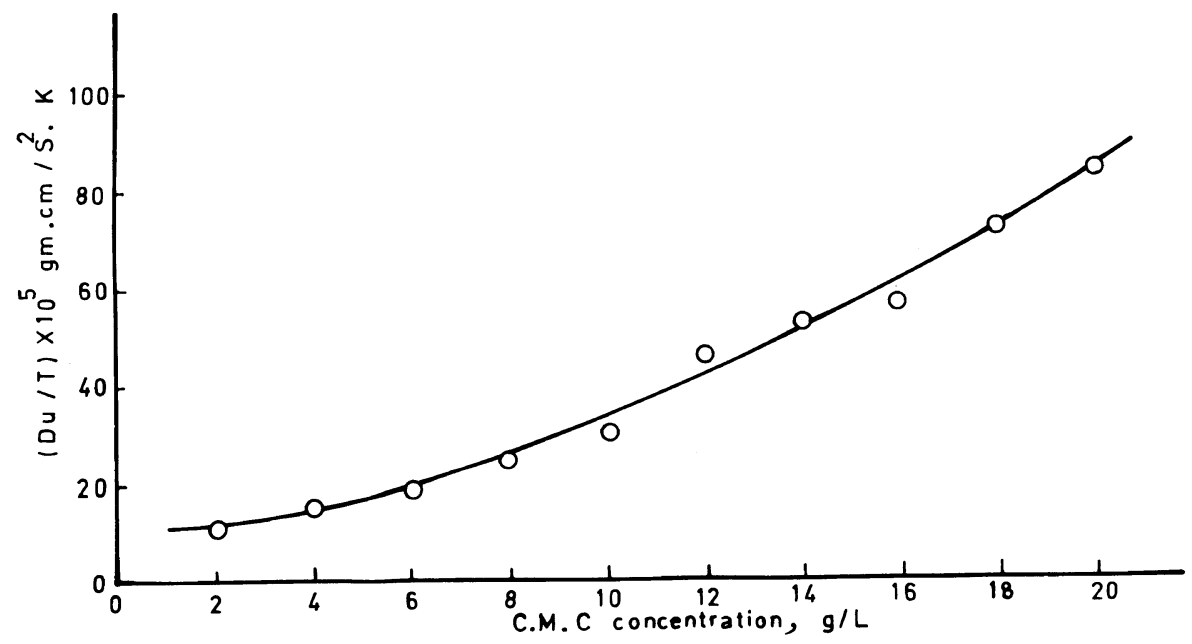

Figure 3. Einstein-Stokes ratio as a function of $\mathrm{CMC}$ concentration. $\mathrm{Na}_{2} \mathrm{SO}_{4}$ concentration $=0.99 \mathrm{M}$; temperature $=25^{\circ} \mathrm{C}$. 
coiled molecule increases solution viscosity to a lower extent. The degree of polymer expansion depends on the ionic strength of the solution, i.e., $\mathrm{CMC}$ concentration and salt concentration; the higher the ionic strength, the lower the ability of the negative segments of the polymer molecule to repel each other to produce a stretched molecule.

iii) CMC molecules are bulky and therefore obstruct the diffusing salt. According to Wang, ${ }^{12}$ the diffusing species has to get around the polymer molecule; i.e., the diffusing path is prolonged in polymer solutions with a consequent delay in the rate of salt diffusion.

iv) CMC is a highly solvated polymer ${ }^{13}$; i.e., a large amount of water is removed from the medium to solvate the polar groups existing in the polymer chain and the counter sodium ions. The removal of this enormous amount of water from the medium decreases the amount of free water available as a solvent. Accordingly, the apparent concentration of the diffusing salt would increase with a consequent increase in the chemical potential of the salt and its diffusivity. The present results as shown in Figures 2,3 show that the fourth effect (hydration of CMC molecules) predominates at relatively low $\mathrm{CMC}$ concentration where salt diffusivity is higher than that in water. The magnitude of the three retarding effects increases with increasing CMC concentration where the salt diffusivity decreases to a value below that in water. Astarita ${ }^{4}$ who studied the diffusion of some nonionic solutes, e.g., allyl alcohol, ethanol and glycerol in CMC solutions found that the presence of $\mathrm{CMC}$ in the medium increases the diffusivity of the solute to a value higher than that in water; this agrees with the present finding. Astarits ${ }^{14}$ reported also that CMC enhances the diffusivity of $\mathrm{CO}_{2}$. Farag et al. ${ }^{15}$ who studied the diffusion of dyes in CMC solution reported increase in the diffusivity of dye molecules in CMC solution. Hansford et al. ${ }^{16}$ who studied the diffusion of benzoic acid in CMC solution reported a decrease in the diffu-

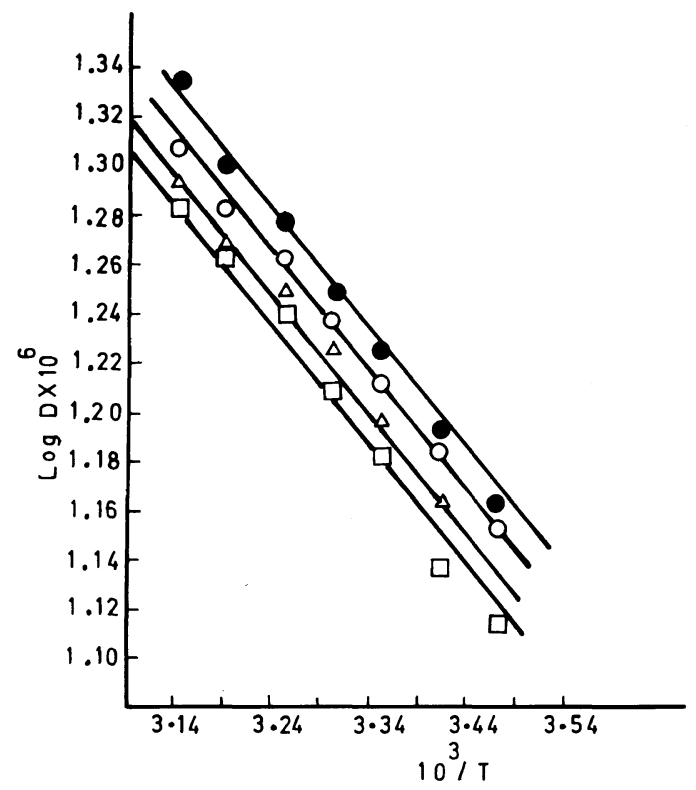

Figure 4. $\log D$ vs. $1 / T$ for the diffusion of sodium sulphate in $\mathrm{CMC}$ solutions. $\mathrm{Na}_{2} \mathrm{SO}_{4}$ concentration $=$ 0.990 M. CMC concentration:, $2 \mathrm{gl}^{-1} ; . \mathrm{O}_{4 \mathrm{~g}^{-1}} ; \triangle$, $6 \mathrm{gl}^{-1} ; \square, 8 \mathrm{gl}^{-1}$.

sivity of benzoic acid in CMC solution. This is probably because Hansford et al. used relatively concentrated non-Newtonian solutions where the enhancing effect of polymer solvation is overshadowed by the retarding effects of the polymer molecules, namely, obstruction, viscosity increase and interionic attraction.

Figure 3 shows the effect of CMC concentration on the Einstein-Stokes ratio

$$
D u / T=\text { constant }
$$

The value of the ratio is not constant but increases with increasing CMC concentration. This confirms the fact that in polyelectrolytes, viscosity is not the sole factor affecting the rate of diffusion. Figure 4 shows the effect of temperature on the diffusivity of sodium sulphate in CMC and the data fit the Arrhenius equation.

$$
D=A \mathrm{e}^{-E / R T}
$$

The activation energy of diffusion $(E)$ is $2600 \mathrm{cal} \mathrm{mol}^{-1}$, Farag et al. who studied the 
diffusion of dyes in CMC solution obtained an activation energy of $5000 \mathrm{cal} \mathrm{mol}^{-1}$. The discrepancy between the value of Farag et al. and the present value may be attributed to the fact that Farag et al. used a bulky organic molecule while the present work used a simple salt.

\section{REFERENCES}

1. R. M. Navari, J. L. Gainer, and K. R. Hall, AIChE J., 17, 1028 (1971).

2. H. R. Osmers and A. B. Metzner, Ind. Eng. Chem. (Fundamentals), 11, 161 (1971)

3. S. U. $\mathrm{Li}$ and J. L. Gainer, Ind. Eng. Chem. (Fundamentals), 7, 433 (1968).

4. G. Astarits, Ind. Eng. Chem. (Fundamentals), 4, 236 (1966).

5. F. T. Wall, P. F. Grieger, and C. W. Childers, J. Am. Chem. Soc., 74, 3562 (1952).

6. F. T. Wall and C. W. Childers, J. Am. Chem. Soc., 75, 3550 (1953).
7. M. Z. El-Abd, G. H. Sedahmed, A. A. Zatout, A. H. Negm, and R. S. Khalil, J. Appl. Chem. Biotechnol. 25, 597 (1975).

8. I. A. S. Mansour, M. Z. El-Abd, and G. H. Sedahmed, J. Appl. Chem. Biotechnol., 28, 854 (1978).

9. G. H. Sedahmed, A. A. Zatout, M. Z. El-Abd, and I. A. S. mansour, Afinidad, 36, 365 (1979).

10. S. E. Tuwiner, "Diffusion and Membrane Technology," Reinhold Publishing Corporation, New York, N. Y., 1962.

11. A. Tager, "Physical Chemistry of Polymers," Mir Publishers, Moscow 1972.

12. H. J. Wang, J. Am. Chem. Soc., 76, 4755 (1954).

13. S. Lifson and J. L. Jackson, J. Chem. Phys., 36, 2410 (1962).

14. G. Astarita, Ind. Eng. Chem. (Fundamentals), 4, 236 (1965).

15. A. A. Farag, H. A. Farag, G. H. Sedahmed, and A. F. El-Nagawy, J. Appl. Polym Sci., 20, 3247 (1976).

16. G. S. Hansford and M. Litt, Paper Presented at the American Institute of Chemical Engineering Meeting, New York, 1967. 